\title{
Deformation defects in nanocrystalline nickel
}

\author{
Xiaolei Wu $\cdot$ En Ma $\cdot$ Y. T. Zhu
}

Received: 28 May 2006/ Accepted: 9 November 2006/ Published online: 3 January 2007

(C) Springer Science+Business Media, LLC 2006

\begin{abstract}
Defects induced by plastic deformation in electrodeposited, fully dense nanocrystalline (nc) $\mathrm{Ni}$ with an average grain size of $25 \mathrm{~nm}$ have been characterized by means of high resolution transmission electron microscopy. The nc Ni was deformed under uniaxial tension at liquid-nitrogen temperature. Trapped full dislocations were observed in the grain interior and near the grain boundaries. In particular, these dislocations preferred to exist in the form of dipoles. Deformation twinning was confirmed in $\mathrm{nc}$ grains and the most proficient mechanism is the heterogeneous nucleation via emission of partial dislocations from the grain boundaries.
\end{abstract}

\section{Introduction}

Nanocrystalline (nc) metals are believed to deform plastically via mechanisms that are fundamentally different from those present in their coarse-grained counterparts [1-12]. Deformation mechanisms sug-

\footnotetext{
X. Wu $(\bowtie)$

State Key Laboratory of Nonlinear Mechanics, Institute of Mechanics, Chinese Academy of Sciences, Beijing 100080, P.R. China

e-mail: xlwu@imech.ac.cn

E. Ma

Department of Materials Science and Engineering, Johns Hopkins University, Baltimore, MD 21218, USA

Y. T. Zhu

Materials Science and Technology Division, Los Alamos

National Laboratory, Los Alamos, NM 87545, USA
}

gested for nc metals include the partial-dislocationmediated processes (PDMPs), e.g., deformation twinning and the formation of extended and full dislocations from the grain boundaries (GBs), GB sliding, and grain rotation.

The mechanisms of plastic deformation have been studied in electrodeposited nc nickel (average grain sizes in the range of 20-30 nm), because $\mathrm{nc} \mathrm{Ni}$ is one of the suitable fcc model metals for such studies. On the experimental side, both in situ and post-mortem transmission electron microscopy (TEM) investigations have been conducted, usually under tensile loading conditions, in order to elucidate the deformation mechanisms [13-16]. The post-mortem observations have failed to reveal deformation debris [13-16]. In contrast, several in situ TEM deformation studies observed dislocation activities [12-15]. The dislocations are described as originating from the GBs and propagating to the adjacent/opposite GB where they are absorbed. This scenario is consistent with an in situ X-ray diffraction study of nc Ni subjected to roomtemperature tensile test, which showed no irreversible peak broadening indicating that defects (dislocations, twins or stacking faults) were not stored at large numbers [5, 17].

However, in situ TEM tensile tests have drawbacks. Spurious deformation processes might be operative, associated with the advancement of a crack tip. Meanwhile, the limited TEM foil thickness can lead to changes in GB structures near the surface and enhance the dislocation activity. Also, only a few grains are sitting on top of each other in a $100 \mathrm{~nm}$ thick foil. So far the observations of dislocation activity are not conclusive, especially since the contrast change during sample pulling can come from other sources. Twinning 
was mentioned to be possible, but only in the last ligament of a thin foil of $\mathrm{nc} \mathrm{Ni}$ during in situ deformation in the TEM [14]. In addition, one in situ dynamic TEM observation of $\mathrm{nc} \mathrm{Ni}$ of grain size $\sim 10 \mathrm{~nm}$ suggested the dominant role of a GB-mediated process, i.e., the individual grain rotation events [12].

Recently, we found using post-mortem TEM observations that the PDMPs do occur in $\mathrm{nc} \mathrm{Ni}$, but require extensive plastic deformation to strains far beyond what was available in a tensile test [18-20]. The PDMPs, including twinning and formation of extended and full dislocations, can also get activated in $\mathrm{nc} \mathrm{Ni}$ during uniaxial tension test at liquid-nitrogen temperature (LNT) $[18,19]$, in addition to severe deformation $[19,20]$. Our results extend the prior evidence in other nc fcc metals, e.g., in $\mathrm{Al}[1,10,21], \mathrm{Cu}$ [22] and $\mathrm{Pd}$ [23, 24], where all the experimental twining evidence were for deformation conditions that resulted in high and complex stresses, owing to high strain rates and/or large plastic deformation leading to saturated defect levels.

Deformation mechanisms in $\mathrm{nc} \mathrm{Ni}$ have also been studied using molecular dynamics (MD) simulations [3, 25]. The slip in nc Ni grains essentially involves the PDMPs, meaning that all dislocation activity is created by the sequential nucleation of single partial dislocation from GBs $[2-4,25,26]$. The generalized planar fault energy (GPFE) curves were proposed to significantly affect the PDMPs [3]. Ni has a high unstable SFE, $\gamma_{\text {usf }}\left(\left(\gamma_{\text {usf }}-\gamma_{\text {usf }}\right) / \gamma_{\text {sf }} \approx 0.4\right.$, and even higher unstable twin fault energy, $\gamma_{\text {utf }}\left(\left(\gamma_{\text {utf }}-\gamma_{\text {sf }}\right) / \gamma_{\text {sf }} \approx 0.9\right.$, which hinder the nucleation of trailing partials and twinning partials. For $\mathrm{nc} \mathrm{Ni}$, therefore, the single leading partial is observed to travel across the entire grain after nucleation, leaving behind an extended stacking fault in the grain [3]. It is noted, however, that this may not be the whole picture in real-world $\mathrm{nc} \mathrm{Ni}$. The emission of a second partial on the same or on an adjacent plane, and therefore the removal of a stacking fault or the nucleation of a twin, is believed to have been missed due to the sub nano-second time restrictions of the MD simulation. [3]. The MD simulations of crack propagation in nc Ni samples have shown extensive dislocation activity at the crack tip, including full dislocations and twinning in grains as small as $10 \mathrm{~nm}$ [27]. This suggests that the dislocation activity observed in in situ TEM tensile testing may be related to the propagation of the crack, and the extrapolation of the results to bulk tensile deformation may be tenuous.

The objective of our work is to conduct postmortem TEM observations of the defect features in an electrodeposited $\mathrm{nc} \mathrm{Ni}$, to shed light on the dislocation processes during uniaxial tension. Our new twist is to carry out the tensile deformation at liquid nitrogen temperature $(77 \mathrm{~K})$, such that some of the deformation defects can be retained. We will demonstrate how the dislocations and twins form and reside in the tiny grains, and their presence in relation to the GBs.

\section{Experimental}

An electrodeposited nc Ni sample was purchased from Goodfellow Inc. The as-received $150 \mu \mathrm{m}$ thick foil was observed to have an average grain size of $\sim 25 \mathrm{~nm}$, similar to those reported before [13, 14, 28-30]. Dogbone shaped tensile samples were prepared using the electrical discharge technique, to gauge dimensions $8 \mathrm{~mm}$ in length and $4 \mathrm{~mm}$ in width. The samples were tested under uniaxial tension to failure at a strain rate of $3 \times 10^{-3} \mathrm{~s}^{-1}$ at liquid-nitrogen temperature $(77 \mathrm{~K})$ by immersing the tensile sample and grips inside liquid nitrogen. The tensile behavior was similar to those published before for electrodeposited nc Ni [28-30]. The strain sustained before failure is approximately $4 \%$, at which point the flow stress (the maximum stress on the respective stress-strain curves) is about 1,500 $\mathrm{MPa}$. The microstructures were examined using a high-resolution TEM (HRTEM), JEM2010F operated at $200 \mathrm{kV}$. TEM samples after tensile tests were prepared as follows. The fractured tensile specimens were mechanically thinned down to about $50 \mu \mathrm{m}$ thick. The slices of $2 \mathrm{~mm}$ long and $1 \mathrm{~mm}$ wide were then cut parallel to the tensile axis. Needle-shaped specimens glued to a metallic ring were produced via electropolishing, followed by Ar ion milling at low temperature (243 K) using a Gatan PIPS with a small incident angle, allowing large, thin and homogeneous areas to be obtained at the tip and edge areas [18].

\section{Results}

Figure 1a is an HRTEM micrograph of an nc grain with a very small grain size of $\sim 15 \mathrm{~nm}$. Two dislocations marked by "Ts" are visible near the GB. Interestingly, these two dislocations form a dipole, i.e., a pair of dislocations close by but with opposite signs of Burgers vector. The inset is an enlarged view of the dislocations. The Burgers circuit from starting point $\mathrm{S}$ to end point $\mathrm{F}$ is drawn to enclose the dislocation core marked by a "T". Clearly, the Burgers vector is one lattice vector, indicative of a full dislocation. Assuming the electron beam and the dislocation line is parallel to [011], the Burgers vector of the 

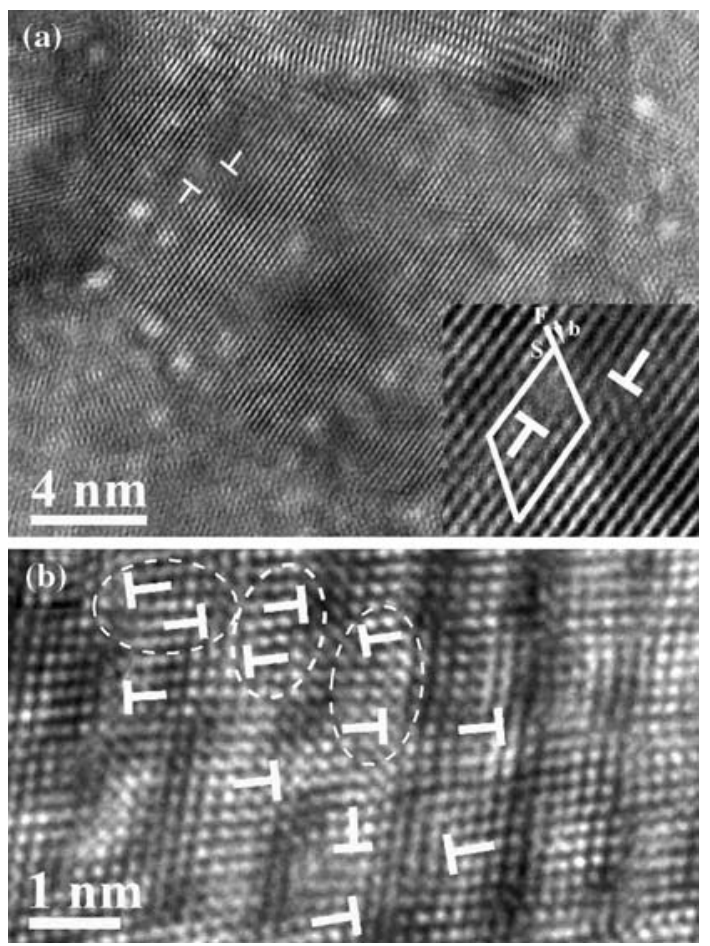

Fig. 1 (a) HRTEM micrograph showing the presence of one dislocation dipole in a nc Ni grain after LNT tensile test. The dislocations are indicated by "Ts". Inset is an enlarged view of dislocation with a Burgers circuit, starting from $S$ and finishing at $\mathrm{F}$, around the dislocation core. (b) HRTEM micrograph showing dislocations on (111) plane of a nc grain of grain size $30 \mathrm{~nm}$. Note that most dislocations form dipoles (some are circled)

dislocation is determined to be $1 / 2[101]$ or $1 / 2[1-10]$, which has an angle of $60^{\circ}$ (or $120^{\circ}$ ) with respect to the dislocation line. For this reason, the dislocation is referred as a $60^{\circ}$ perfect dislocation. The $60^{\circ}$ type dislocations, often observed in deformed FCC materials, are glissible along the (111) slip planes.

We further observed the non-uniformly distributed dislocations that indicate preferential deformation and defect storage in the vicinity of GBs, as shown in Fig. 1b. Figure $1 b$ is an HRTEM image, showing the presence of dislocations just in a localized area near the $\mathrm{GB}$ of a larger nc grain of $\sim 30 \mathrm{~nm}$. All dislocations are $60^{\circ}$ type. This demonstrates that the plastic deformation varies from place to place in the nc grain probably due to highly heterogeneous nature. Hence, the dislocations of high density are only infrequently observed and exist locally in the grain interior. Importantly, the dislocations occur mostly in the form of dislocation dipoles (some are circled). The formation reason of the dipoles and their effect on plastic deformation of nc grains is still unknown. We examined both as-received samples and those pulled to failure after RT tensile tests. In both cases, almost no full dislocations were observed in the nc grains. Hence, the dislocations in Fig. 1 are probably formed due to plastic deformation at $77 \mathrm{~K}$.

We also observed the relatively clean interior in many grains (more than half of the grains investigated), i.e., no dislocations and twins. This is understandable in three respects. First, even though FCC metals have 12 slip systems, the local single slip probably dominates in $\mathrm{nc} \mathrm{Ni}$ grains during deformation especially at low temperatures [31]. It is considered that at low temperatures one slip system is strongly preferred due to intrinsic features of the activation process itself [32, 33]. Hence, dislocation will be observed only if the direction of electron beam is proper to show dislocations in the activated slip planes. Unfortunately, the tilting operation in a HRTEM is limited. Second, these "clean" nc grains may experience another kind of plastic deformation as predicted by the MD simulations [3]. After the formation of extended stacking faults via the emission of (leading) partials from the $\mathrm{GB}$, the trailing partial erases the stacking fault as it runs across the grain to reach the opposite GB, leaving no footprint for detection in post-mortem HRTEM [5]. Third, these "clean" nc grains may indeed do not experience any plastic deformation.

Figure 2a is an HRTEM image showing a nc grain in which twinning occurs. The arrow indicates the twin boundary. The details of the twin are shown in Fig. 2 b.
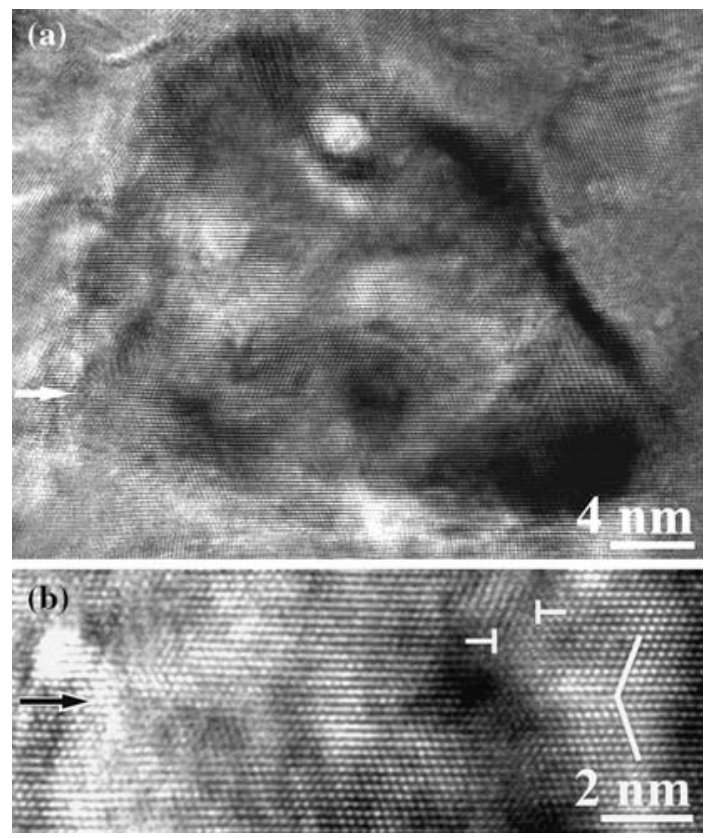

Fig. 2 (a) HRTEM image showing a nc grain with its lower part transforming into a twin. The arrow indicates the twin boundary. (b) Enlarged view showing the details of the twin. Note the presence of a dislocation dipole near the twin boundary 
The twin may nucleate heterogeneously at the GB and grow into the grain interior via partial dislocation emission from the GB $[2,10,18,19]$. We found many such examples in our $\mathrm{nc}-\mathrm{Ni}$, indicating the dominant role of this mechanism in initiating twinning [18]. It is also noted that a dislocation dipole exists much near the twin boundary. Based on extensive HRTEM observations, the pre-existing growth twins were few and far between in the 100 grains we examined, consistent with the previous observations [13]. We also examined samples pulled to failure after RT tensile tests. Again we only observed very few twins (no more than 5 in $~ 120$ grains). Some of the twins observed may be growth twins. This finding is consistent with the published X-ray peak broadening results [5]. However, after LNT tension, the twins were observed. Hence, we concluded that the observed twins were deformation twins produced by plastic deformation at low temperatures.

Two twinning systems were also found infrequently in operation in a single grain. This scenario is shown in Fig. 3a, where the two sets of deformation twins on two different $\{111\}$ planes are marked. In addition, multifold twins are occasionally observed, a typical example is shown in Fig. 3b, an HRTEM image showing a threefold twin. This twin was likely heterogeneously

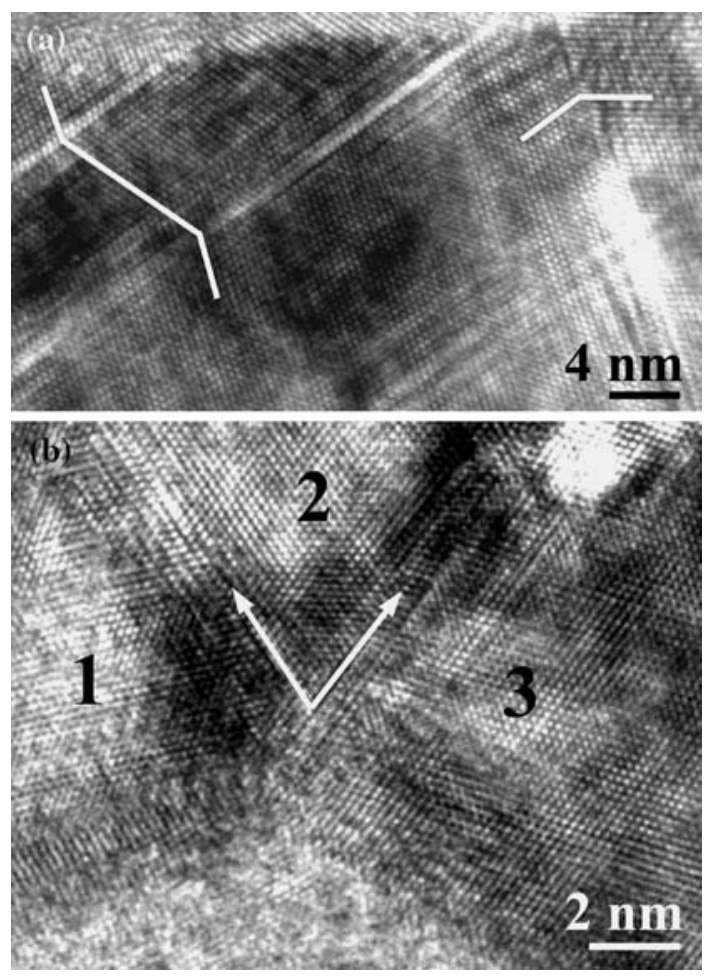

Fig. 3 HRTEM images showing (a) two twinning systems and (b) threefold twin. The twin boundary is indicated by arrows and each twin domain is numbered (1-3) formed via the emission of Shockley partial dislocations from GBs, much like the fivefold twin reported in $\mathrm{nc} \mathrm{Cu}$ after severe deformation [22]. The formation mechanism of multifold deformation twins is likely due to sequential twinning [34]. Deformation twins observed in $\mathrm{nc} \mathrm{Ni}$ grains indicates the operation of PDMPs.

Figure 4 is an HRTEM micrograph showing lattice structure close to the GB of a nc grain of $\sim 30 \mathrm{~nm}$ in size. Dislocations are visible near the GB. These dislocations indicate a single slip system. Interestingly, a micro-twin is also seen simultaneously, as indicated by the arrow on the right. This is, in fact, a twin nucleus with a thickness of only two atomic planes. Such a twin was formed by the dynamic overlapping of two extended partial dislocations with SFs on adjacent slip planes [21, 26]. This mechanism occurs when the concentration of the extended dislocations inside the grain is high. The growth of twin nucleus (with more atomic planes) may be realized by adding more SFs on either side of the twin and shown in Ref. [19].

The localized severe lattice distortion is also observed in the vicinity of the GB. It is seen that the GB region has a misorientation angle of $10^{\circ}$ from the grain interior, as marked by two lines. Dislocations are found to preferentially locate in the GB region, examples of which are indicated by "Ts". It is possible that dislocations are emitted from the GB of the nc grain. The dislocations were stopped and pinned when moving into the grain interior, leading to a localized heavy deformation region. The 2-D crystal orientation in the adjacent grain is visible, indicative of a high angle GB between these two grains. This high angle GB is nearly edge-on and not overlapped, without the presence of the periodic Moiré Fringes at/near the GB. Hence, the orientation change in the localized deformation region should not be an artifact due to

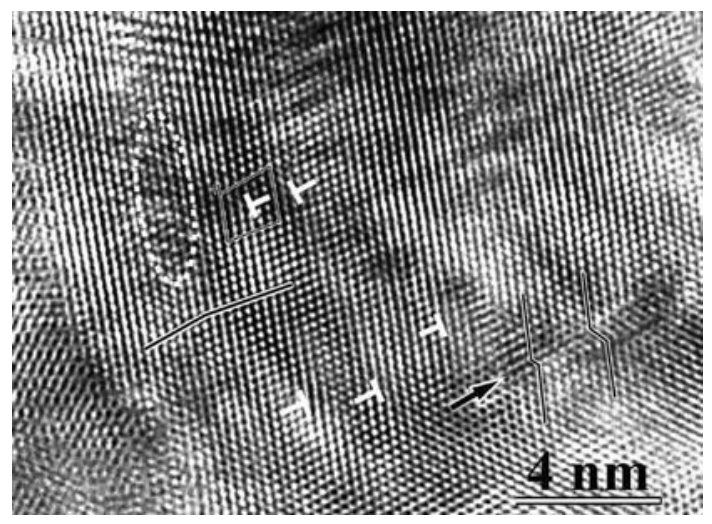

Fig. 4 HRTEM images showing the co-existence of full dislocations and a microtwin indicated by an arrow. Also note the presence of local severe lattice distortion (circled) 
slightly overlapped two nano-grains in the vicinity of the GB.

\section{Discussion}

We examined both as-received samples and those pulled to failure after tensile tests at the room temperature [18]. In both cases, almost no full dislocations were observed in the nc grains. After uniaxial tension at low temperature $(77 \mathrm{~K}, \sim 4 \%$ strain) the dislocations form. However, there are still many grains (more than half of the grains investigated) with relatively clean interior (i.e., no dislocations and twins). We also observed the non-uniformly distributed dislocations that indicate preferential deformation and defect storage in the vicinity of GB. The presence of trapped full dislocations (shown in Figs. 1, 2, 4) is a significant deformation feature in $\mathrm{nc} \mathrm{Ni}$ during uniaxial tension at $77 \mathrm{~K}$. More specifically, the plastic deformation may vary from grain to grain and even from place to place in the grain interior, concentrating more in regions near the GB. These results demonstrate the inhomogeneous nature of the deformation pattern within the nc grain. These dislocations may be directly emitted from the GBs. They may also form in the way suggested by MD simulations [3]: after a single partial dislocation is nucleated, the nucleation of a trailing partial dislocation follows on the same slip plane, and as such a full dislocation will be formed when the trailing partial catches up with the leading one. The dislocation processes in nc grains are thermally activated, as revealed by the stronger-than-normal temperature/ strain rate dependence of the flow stresses [28-30, 3436]. At low deformation temperature, the relaxation/ adjustment in the grains and GBs is depressed, and the activation of dislocations would then rely largely on the local stress intensity (depending on the applied stresses, internal stresses, and the stress field of the leading partial). Their population becomes considerable after tensile tests at $77 \mathrm{~K}$, as the reduced thermal mobility renders pinning more likely, e.g., by GBs along which the two ends of the dislocation glide. The mechanisms for the pinning of dislocations include impurities, formation of locks [26] or dislocation jogs [36].

We note that the formation of dislocation dipoles seems to be frequent. Due to the shape of the stress field around a dislocation, the configuration of dipoles shown, for example in Fig. 1, will allow the most overlap of stress fields and cancel out the stresses. However, the formation mechanism of dipoles in $\mathrm{nc}$ grains needs further investigations.
Another main defect configuration is deformation twins, as shown in Figs. 2, 3. Previous tensile tests at RT did not produce evidence of twins. In such tests, the samples usually sustain a small plastic strain $(\sim 4 \%)$ in tension before failure. The initial portion of the stress-strain curve involved apparent bending [29, 30]. This may be related to the exhaustion of dislocation sources that are pre-existing, or GB segments that are relatively easy to shear/slide. Twinning was apparently not yet competitive with other possible means that can produce the plastic strain. Unfortunately, further tensile straining was not possible beyond the point where the (necking) instability set in. The deformation flow stress observed in tensile test at low temperatures is higher than that at room temperature with strain of approximately $4 \%$, and there can be large variations of local stresses, at least more non-uniform than the room temperature case. These allow the various PDMPs to become competitive. The higher stress at low temperatures produces larger difference in the resolved shear stress between the leading and trailing partials, which have different Schmid factors due to their orientation difference. This further reduces the probability of the trailing partial and favors twin nucleation because partials have the same Burgers vector like the leading partial. Thus, twinning becomes more favorable at low temperatures. In addition, the local stress concentration is a crucial factor in determining the type of dislocation process activated [38]. When the applied stress is sufficiently large, slip mechanisms and multiple twinning events are active for nc Ni.

\section{Summary}

We have investigated the post-mortem deformation microstructure in nanocrystalline $\mathrm{Ni}$ after uniaxial tension at liquid-nitrogen temperature ( $\sim \%$ strain). The trapped full dislocations, mainly in the form of dipoles, are observed in the grain interior and especially near the grain boundaries. Deformation twinning was confirmed to operate in nc grains. It is suggested that when $\mathrm{nc} \mathrm{Ni}$ is forced to deform at high stresses, local stress concentrations will inevitably build-up to sufficient levels to activate slip mechanisms and deformation twinning as competitive deformation responses.

Acknowledgements XLW was supported by Natural Science Foundation of China (50471086, 50571110, 10472117), 973 Project of China (2004CB619305), Key Project of CAS. 


\section{References}

1. Chen MW, Ma E, Hemker KJ, Sheng HW, Wang YM, Cheng X (2003) Science 300:1275

2. Yamakov V, Wolf D, Phillpot SR, Mukherjee AK, Gleiter H (2004) Nature Mater 3:43

3. Van Swygenhoven H, Derlet PM, Frøseth AG (2004) Nature Mater 3:399

4. Wolf D, Yamakov V, Phillpot SR, Mukherjee AK, Gleiter AH (2005) Acta Mater 53:1

5. Budrovic Z, Van Swygenhoven H, Derlet PM, Van Petegem S, Schmitt B (2004) Science 304:273

6. Kumar KS, Van Swygenhoven H, Suresh S (2003) Acta Mater 51:5743

7. Wang YM, Ma E (2004) Acta Mater 52:1699

8. Ma E (2006) JOM 58(4):49; Met Mater Int 10 (2004) 527

9. Meyers MA, Mishra A, Benson DJ (2006) Prog Mater Sci 51:427

10. Liao XZ, Zhou F, Lavernia EJ, Srinivasan SG, Baskes MI, He DW, Zhu YT (2003) Appl Phys Lett 83:632

11. Zhu YT, Langdon TG (2005) Mater Sci Eng A 409:234

12. Shan Z, Stach EA, Wiezorek JMK, Knapp JA, Follstaedt DM, Mao SX (2004) Science 305:654

13. Dalla Forre F, Van Swygenhoven H, Victoria M (2002) Acta Mater 50:3957

14. Kumar KS, Suresh S, Chisholm MF, Horton JA, Wang P (2003) Acta Mater 51:387

15. Hugo RC, Kung H, Weertman JR, Mitra R, Knapp JA, Follstaedt DM (2003) Acta Mater 51:1937

16. Legros M, Elliott BR, Rittner MN, Weertman JR, Hemker KJ (2000) Philos Magn A 80:1017

17. Brandstetter S, Budrovic Z, Van Petegem S, Schmitt B, Stergar E, Derlet PM, Van Swygenhoven H (2005) Appl Phys Lett 87:231910

18. Wu XL, Zhu YT, Chen MW, Ma E (2006) Scr Mater 54:1685

19. Wu XL (2006) Appl Phys Lett 88:061905
20. Ma E (2006) Appl Phys Lett 88:231911

21. Wu XL, Zhu YT, Ma E (2006) Appl Phys Lett 88:121905

22. Liao XZ, Zhou F, Lavernia EJ, He DW, Zhu YT (2003) Appl Phys Lett 83:5062

23. Liao XZ, Zhao YH, Srinivasan SG, Zhu YT, Valiev RZ, Gunderov DV (2004) Appl Phys Lett 84:592

24. Rosner H, Markmann J, Weissmuller J (2004) Philos Magn Lett 84:321

25. Markmann J, Bunzel P, Rösner H, Liu KW, Padmanabhan KA, Birringer R, Gleiter H, Weissmüller J (2003) Scr Mater 49:637

26. Froseth AG, Derlet PM, Van Swygenhoven H (2004) Acta Mater 52:5863

27. Yamakov V, Wolf D, Phillpot SR, Gleiter H (2002) Acta Mater 50:5005

28. Farkas D, Van Petegem S, Derlet PM, Van Swygenhoven H (2005) Acta Mater 53:3115

29. Wang YM, Cheng S, Wei QM, Ma E, Nieh TG, Hamza A (2004) Scr Mater 51:1023

30. Wang YM, Hamza A, Ma E (2005) Appl Phys Lett 86:241917

31. Wang YM, Ma E (2004) Appl Phys Lett 85:2750

32. Hollang L, Hieckmann E, Brunner D, Holste C, Skrotzki W (2006) Mater Sci Eng A 424(1-2):138

33. Cahn JW, Nabarro FRN (2001) Philos Magn A81:1409

34. Essmann U, Rapp M, Wilkens M (1968) Acta Metall 16:1275

35. Zhu YT, Liao XZ, Valiev RZ (2005) Appl Phys Lett 86:103112

36. Zhu YT, Liao XZ, Zhao YH, Srinivasan SG, Zhou F, Lavernia EJ (2004) Appl Phys Lett 85:5049

37. Wei Q, Cheng S, Ramesh KT, Ma E (2004) Mater Sci Eng A381:71

38. Liao XZ, Srinivasan SG, Zhao YH, Baskes MI, Zhu YT, Zhou F, Lavernia EJ, Xu H (2004) Appl Phys Lett 84:3564

39. Froseth AG, Derlet PM, Van Swygenhoven H (2005) Adv Eng Mater 7:16 